\title{
Spray-Drying: An Emerging Technique for Pharmaceutical Product Development
}

\section{Jagadevappa Patil}

VT's Shivajirao S Jondhle College of Pharmacy, Asangaon - 412 601, Thane, Maharashtra, India

"Corresponding author: Jagadevappa Patil, VT's Shivajirao S Jondhle College of Pharmacy, Asangaon-412 601, Thane, Maharashtra, India, Tel: 9448816812; E-mail: pharmajspatil@gmail.com

Received date: March 10, 2016; Accepted date: March 14, 2016; Published date: March 18, 2016

Copyright: (c) 2016 Patil J. This is an open-access article distributed under the terms of the Creative Commons Attribution License, which permits unrestricted use, distribution, and reproduction in any medium, provided the original author and source are credited.

\section{Introduction}

Atomization of a solution of one or more solutes through a nozzle, spinning disk or other device followed by flash evaporation of the solvent from the droplets is termed as spray drying [1]. Spray-drying is a simple and robust continuous unit operation process which converts liquid to solid powder [2]. Thus, this process is based on drying of atomized liquid stream by contacting with high temperature gas stream by evaporation of liquid from the droplets, forming particles [3]. Being one of the oldest drying methods although it was known and used much before that, spray-drying gained much importance during the Second World War with the sudden need to reduce the transport weight of the foods and other materials [4]. Spray-drying is a versatile and one of the most emerging technologies for the pharmaceutical industry especially for novel pharmaceutical product development. This ideal technique is known to give up final product which complies with precise quality standards with respect to particle size and their distribution, residual solvent / moisture content, morphology and bulk density. Spray-drying offers multiple opportunities to provide very fine particles for pulmonary / nasal delivery, big agglomerated powers for oral administration, amorphous to crystalline products and is potential for one step formulation that no other technologies can offer. Apart from its ability to have high precision control over the particle size, bulk density, degree of crystallinity and residual solvent / moisture content in final product, this technique also holds good for typical applications in pre-formulated products, microencapsulation, solid solutions, improved bioavailability and stability through inclusion complex system and also provide opportunity to process the products with unusual or difficult characteristics such as sticky or hygroscopic products, slowly crystallizing products, products which are difficult to isolate and thermo sensitive materials.

\section{Atmospheric Spray Freeze-Drying (ASFD)}

The main purpose of manufacturing dry powder pharmaceuticals is to increase stability and shelf life of the formulations storing at room temperature, reducing the bulk and weight, and facilitating the development of novel self-administrative delivery systems such as dry powder inhalers and nasal sprays for pulmonary administration. Basically, conversion of liquid into solid is achieved through lyophilization or freeze-drying, which involves freezing a bulk liquid solution and subliming off the solvent using temperature and vacuum. Although lyophilization is slow and energy consuming method which often require post processing to produce a powdered product suitable for its end-use application, most pharmaceutical companies have used this technique for decades. To increase the manufacturing efficiency and achieve higher activity and longer shelf lives of complex and fragile dry powder biologics, pharmaceutical companies are under pressure due to limitations of lyophilization technique in bulk processing. In order to enhance the reliability and performance of existing lyophilization technique, scientist and engineer offer a dashboard of opportunity to fabricate obscure features and properties into each particle. Engineered dry powdered pharmaceutical products can be prepared via one of the advanced technologies is atmospheric spray freeze-drying (ASFD) technique which is a hybrid process of both lyophilization and traditional spray-drying without many of the limitations associated with lyophilization alone. In this process, a liquid formulation is atomized and the individual droplets are rapidly cooled to suspend solute streaming and molecular motion, and lock in the spherical droplet shape. The frozen particles are then dried in a cold, dry gas flow to the desired, predetermined low moisture content. Particles thus obtained rare porous, spherical and aerodynamically light making them ideal for a variety of end-use applications.

\section{Applications of spray-drying in pharmaceuticals and drug delivery}

Spray-drying offers multiple applications in pharmaceutical and drug delivery filed, few of the important are briefed here.

1. Excipient production: Spry-drying can be effectively exploited in the production of pharmaceutical excipients with desired properties. For example, directly compressible powders in solid dosage forms such as tablets with improved compressibility.

2. Encapsulation: Microcapsules and nanoparticles with therapeutic core and biocompatible coat material are successfully prepared by spray-drying technique to develop novel controlled drug delivery systems. Patil et al. [5,6] have reported the microencapsulation of antiretroviral drug in olibanum gum resin and encapsulation of rifampicin in same olibanum gum resin by novel spray-drying technique $[5,6]$.

3. Enhancing bioavailability: Spray drying can be used to enhance the solubility and dissolution rate of poorly soluble drugs. This usually occurs via the formation of pharmaceutical inclusion complexes or via the development of solid dispersions [7-10].

4. Dry powder inhalers: Spray drying is an excellent method for the fabrication of dry powder inhaler formulations since particle size distribution and residual moisture content of the spray dried powders can be easily controlled by the process conditions. The systemic pulmonary delivery of rifampicin in rats was reported with high absorption and improved absolute bioavailability relative to oral administration [11].

5. Drying of heat sensitive materials: In addition, the processing of heat sensitive materials by spray-drying is feasible due to the cooling effect caused by the flash solvent evaporation. Hence, the actual temperature of the dried product is far below the outlet 
temperature of the drying air. At higher inlet drying air temperature, the resulting lower residual moisture content of the spray dried powder provided excellent storage properties, although more protein inactivation occurred [12].

\section{Conclusion}

For the successful delivery of sophisticated therapeutics and their ultimate benefits putting pressure and offering the opportunities to pharmaceutical industries for the development of new technologies that can answer the problems. The enormous growth in therapeutics obtained from biotechnology and relocation of existing drugs into novel efficient delivery modes are testament to the changing scenario. Spray drying is a versatile manufacturing technique for the pharmaceutical industry since it uses a one-step process for formation and drying of powders. Spray drying has a multiple applications in the pharmaceutical and biotech industry to improve the compressibility, to perform microencapsulation, granulation and complex formation. In addition, spray drying is successfully used for the modification of biopharmaceutical properties.

\section{References}

1. Connor REO, Felton LA, Schwartz JB (2005) Remmington the Science and Practice of Pharmacy, Powders, David Troy (21stedn), Lippincott Williams and Wilkins, Baltimore, Philadelphia.

2. Spray Drying - scale up from research to industrial production.

3. Shabde V (2006) Optimal Design and Control of a Spray Drying Process that Manufactures Hollow Micro - Particles.

4. www.pharminfo.net
5. Patil JS, Kadam DV, Shiralshetti SS, Marapur SC, Kamalapur MV (2012) Utilization of Natural Olibanum gum Resin as a Rate Controlling Polymer in Design and Evaluation of Microspheres of an Antiretroviral Drug by Using a Unique Spray - drying Technique. Indian J Pharm Edu Res 46: 155-160.

6. Patil JS, Jagadhane VB, Jamagondi LN, Gurav PB (2014) Rifampicin Loaded Spray-dried Olibanum gum Resin Pulmospheres for Lung Delivery. Special Issue on: Drug Delivery Using Nanomedicine and Nanotechnology. J Drug Deliv Therapeut 4: 15-20.

7. Patil JS, Sarasija S (2009) Physicochemical Characterization, in vitro Release and Permeation Studies of Respirable Rifampicin-cyclodextrin Inclusion Complexes. Indian J Pham Sci 71: 638-643.

8. Patil JS, Pandya NR, Marapur SC, Shiralshetti SS (2010) Influence of Method of Preparation on Physicochemical Properties and in vitro Drug Release Profile of Nimodipine-cyclodextrin Inclusion Complexes: A Comparative Study. Int J Pharm Pharm Sci 2: 71-81.

9. Shiralshetti SS, Patil AS, Patil JS (2010) Influence of Method of Preparation on Physicochemical Properties and in vitro Drug Release Profile of Simvastatin-cyclodextrin Inclusion Complexes: A Comparative Study. Int J Chem Tech Res 2: 562-571.

10. Shiralshetti SS, Patil AS, Patil JS (2010) Influence of Method of Preparation on Physicochemical Properties and in vitro Drug Release Profile of Simvastatin - cyclodextrin Inclusion Complexes: A Comparative Study. Int J Curr Pharm Res 2: 7-12.

11. Patil JS, Kusum Devi, Kshama Devi, Sarasija S (2015) Formulation and Evaluation of Novel Spray-dried Alginate Microspheres as Pulmonary Delivery Systems of Rifampicin in Rats. Indian J Pharm Edu Res 49: 320-328.

12. Adler M, Lee G (1999) Stability and surface activity of lactate dehydrogenase in spray-dried trehalose. J Pharm Sci 88: 199-208. 